\title{
Theoretical Limits and a Practical Estimator for Joint Estimation of Respiration and Heartbeat Rates Using UWB Impulse Radio
}

\author{
Sinan Gezici, Member, IEEE, and Orhan Arikan, Member, IEEE
}

\begin{abstract}
In this paper, Cramer-Rao lower bounds are derived for joint estimation of respiration and heartbeat rates via impulse radio ultra-wideband signals. Generic models are employed for displacement functions due to respiration and heartbeat, and the bounds are obtained for the cases of known and unknown chamnel coefficients. In addition, a two-step suboptimal estimator is proposed, which is based on joint time-delay estimation followed by a least-squares approach. It is shown that the proposed estimator is asymptotically optimal under mild conditions. Simulation studies are performed to evaluate the lower bounds and performance of the proposed estimator for realistic system parameters.
\end{abstract}

Index Terms-Ultra-wideband (UWB), impulse radio (IR), Cramer-Rao lower bound (CRLB), least-squares (LS) estimation.

\section{INTRODUCTION}

After the US Federal Communications Commission's (FCC's) approval on the limited use of ultra-wideband (UWB) technology in February 2002 [1], communications and imaging systems that employ UWB signals have drawn considerable attention. Large bandwidths of UWB signals not only facilitate high-speed data transmission, but also result in high time resolution, which important for accurate ranging and location estimation [2]. Commonly, impulse radio (IR) systems, which transmit very short duration pulses with a low duty cycle, are employed to implement UWB systems [3]. In an IR communications system, a train of pulses is sent and information is usually conveyed by the positions or the amplitudes of the pulses. In addition to communications applications, IR-UWB systems have also been considered for medical applications [4]-[8]. Specifically, high spatial resolution of UWB signals facilitates detection of objects and estimation of vital signal parameters, such as respiration and heartbeat rates of humans, in a given environment. Estimation of vital signal parameters can be very important in many scenarios, including searching people under debris after an earthquake, through-the-wall health monitoring of hostages, and non-invasive patient monitoring [4].

Compared to the Doppler based techniques for estimation of vital signal parameters [9], [10], IR-UWB signaling has advantages such as high penetration capability, which facilitates through-the-wall applications, and low transmission power. In [5], possible medical applications of UWB signals are addressed, and their penetration and reflection properties are investigated. The channel characteristics for the signals reflecting from a human chest are studied in [6], which also presents an algorithm for respiration rate estimation. In [8],

\footnotetext{
${ }^{0}$ The authors are with the Department of Electrical and Electronics Engineering, Bilkent University, Bilkent, Ankara TR-06800, Turkey, email: \{gezici,oarikan\} (cee.bikent.edu.tr
}

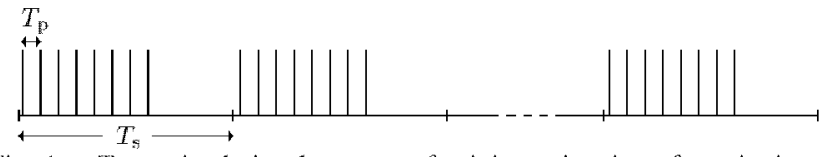

Fig. 1. Transmitted signal structure for joint estimation of respiration and heartbeat parameters.

respiratory detection of hidden humans using UWB signals is implemented. A mathematical framework for estimation of vital signal parameters is established in [4], which employs the Fourier transform and motions filters for estimation of respiration and heartbeat rates.

In [7], theoretical limits for estimation of vital signal parameters are derived under the assumption that there is one periodically moving object in the environment. Although this model can be employed for estimation of respiration rate parameters ${ }^{1}$, joint estimation of heartbeat and respiration rates requires an extended analysis, which takes both respiration and heartbeat related parameters into account. In this paper, we derive Cramer-Rao lower bounds (CRLBs) for joint estimation of respiration and heartbeat parameters, and propose an asymptotically optimal estimator (under certain conditions), which is composed of time-delay and least-squares (LS) estimators. We numerically evaluate the CRLBs for practical systems, and compare the performance of the proposed estimator with the derived CRLBS.

The remainder of the paper is organized as follows. In Section II, the signal model used for parameter estimation is presented. In Section III, the maximum likelihood (ML) solution is obtained and CRLBs are derived for generic time displacement functions. In Section IV, a two-step estimator based on time-delay and LS estimation is proposed and its optimality properties are investigated. Then, numerical examples and simulation results are presented in Section $\mathrm{V}$, and concluding remarks are made in Section VI.

\section{Signal Model}

Consider a sequence of pulse bursts as shown in Figure 1, which is mathematically expressed as

$$
s(t)=\frac{1}{\sqrt{N}} \sum_{k=0}^{N-1} w\left(t-k T_{\mathrm{s}}\right),
$$

where $N$ is the number of bursts, $T_{\mathrm{s}}$ is the burst period, and $w(t)$ is a burst of pulses, which consists of $M$ pulses and is

Since reflections from a heart are much weaker than those from a human chest, the respiration rate estimation problem can omit the signals reflecting from the heart; hence, it can be studied in the framework proposed in [7]. 
given by

$$
w(t)=\sum_{j=0}^{M-1} p\left(t-j T_{\mathrm{p}}\right)
$$

with $p(t)$ denoting the transmitted pulse and $T_{\mathrm{p}}$ being the interval between consecutive pulses. It is assumed that $T_{\mathrm{p}}>$ $T_{\mathrm{W}}$ where $T_{\mathrm{w}}$ denotes the width of $p(t)$.

The burst of pulses in (1) is aimed at an object being monitored and reflections are collected by a receiver. Pulses in each burst are employed to obtain a reliable channel profile (i.e., to improve signal-to-noise ratio (SNR)), and comparison of channel profiles obtained from consecutive bursts is used to estimate certain parameters of the object in the environment.

In this paper, it is assumed that the received signal consists of two signal components (reflections), one due to respiration and the other due to heartbeat, and additive white Gaussian noise; i.e.,

$$
\begin{aligned}
r(t)=\frac{1}{\sqrt{N}} & \sum_{k=0}^{N-1}\left[\alpha_{\mathrm{b}} w\left(t-k T_{\mathrm{s}}-g_{k}\left(\boldsymbol{\theta}_{\mathrm{b}}\right)\right)\right. \\
& \left.+\alpha_{\mathrm{h}} w\left(t-k T_{\mathrm{s}}-h_{k}\left(\boldsymbol{\theta}_{\mathrm{h}}\right)\right)\right]+\sigma n(t),
\end{aligned}
$$

where $n(t)$ denotes zero-mean white Gaussian noise with unit spectral density, $\alpha_{\mathrm{b}}$ and $\alpha_{\mathrm{h}}$ are channel coefficients for the respiration (breathing) and heartheat signal components, respectively, and $g_{k}\left(\boldsymbol{\theta}_{b}\right)$ and $h_{k}\left(\boldsymbol{\theta}_{\mathrm{h}}\right)$ represent, respectively, the time displacement functions induced by respiration and heartbeat of the person being monitored. Note that the unknown parameters of the displacement function $g_{k}\left(\boldsymbol{\theta}_{\mathrm{b}}\right)$ are denoted by $\theta_{\mathrm{b}}$, which consists of $K_{1}$ scalar parameters; i.e., $\theta_{\mathrm{b}}=\left[\theta_{\mathrm{b}, 1} \cdots \theta_{\mathrm{b}, K_{1}}\right]$. Similarly, the unknown parameters of the displacement function $h_{k}\left(\boldsymbol{\theta}_{\mathrm{h}}\right)$ are given by $\boldsymbol{\theta}_{\mathrm{h}}=$ $\left[\theta_{\mathrm{h}, 1} \cdots \theta_{\mathrm{h}, K_{2}}\right]$. For example, if the displacement function for the respiration is a sinusoidal function [6], $g_{k}\left(\boldsymbol{\theta}_{\mathrm{b}}\right)$ becomes

$$
g_{k}\left(\boldsymbol{\theta}_{\mathrm{b}}\right)=\tau_{\mathrm{b}}+A_{\mathrm{b}} \sin \left(2 \pi f_{\mathrm{b}} k T_{\mathrm{s}}+\phi_{\mathrm{b}}\right),
$$

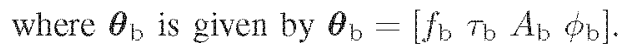

Since the signal component reflecting from the heart travels a longer distance than the one reflecting from the chest, we assume that $h_{k}\left(\theta_{\mathrm{h}}\right)>g_{k}\left(\theta_{\mathrm{b}}\right) \forall k$. We also assume that the range of the displacement functions and the pulse $p(t)$ satisfy $(M-1) T_{\mathrm{p}}+T_{\mathrm{w}}+\max _{k, l}\left\{h_{\bar{k}}\left(\boldsymbol{\theta}_{\mathrm{h}}\right)-g_{l}\left(\boldsymbol{\theta}_{\mathrm{b}}\right)\right\}<T_{\mathrm{s}}$, so that there is no overlap between consecutive pulse bursts, which is usually the case in practice.

Note that the signal model in (3) is not very realistic for wideband pulse-based systems, since it ignores the multipath components from the other objects in the environment. However, it is still an important first step towards understanding of a real system as the main ideas in the analysis can be extended to multipath scenarios. Also, this model gets more accurate when directional antennas are used for transmission and reception, and/or an efficient clutter removal algorithm [11] is applied before the parameter estimation process. Finally, the theoretical limits on the accuracy of parameter estimation obtained using the signal model in (3) provides a lower bound for the more general multipath case as well, since more misance parameters exist for the latter.

\section{MaXimum LikeliHood SOLUTION AND CRLB Calculations}

Let the unknown parameters of the received signal in (3) be represented as

$$
\lambda=\left[\begin{array}{llll}
\theta_{b} & \theta_{\mathrm{h}} & \alpha_{\mathrm{b}} & \alpha_{\mathrm{h}}
\end{array}\right],
$$

which has $K\left(=K_{1}+K_{2}+2\right)$ components. Let $\tilde{r}_{\lambda}(t)$ represent the received signal in the absence of noise; i.e., $r(t)=\tilde{r}_{\lambda}(t)+$ $\sigma n(t)$. Then, the log-likelihood function of $\lambda$ can be expressed as [7]

$$
\Lambda(\lambda)=c-\frac{1}{2 \sigma^{2}} \int_{0}^{T}\left|r(t)-\tilde{r}_{\lambda}(t)\right|^{2} d t
$$

where $c$ denotes a constant independent of $\lambda$, and $T=N T_{\mathrm{s}}$ is the observation interval.

Considering real signals, the ML solution can be obtained from (6) as

$$
\hat{\boldsymbol{\lambda}}_{\mathrm{ML}}=\arg \max _{\lambda} \int_{0}^{T}\left[2 r(t) \tilde{r}_{\lambda}(t)-\left(\tilde{r}_{\lambda}(t)\right)^{2}\right] d t
$$

which requires an exhaustive search over the parameter space for $\lambda$.

From (6), the components of the Fisher information matrix (FIM) I can be obtained as follows [12], [7]:

$$
[\mathbf{I}]_{i i}=\mathrm{E}\left\{\left(\frac{\partial \Lambda(\lambda)}{\partial \lambda_{i}}\right)^{2}\right\}=\frac{1}{\sigma^{2}} \int_{0}^{T}\left(\frac{\partial \tilde{r}_{\lambda}(t)}{\partial \lambda_{i}}\right)^{2} d t
$$

for $i=1, \ldots, K$, and

$$
[\mathbf{I}]_{i j}=\mathrm{E}\left\{\frac{\partial \Lambda(\boldsymbol{\lambda})}{\partial \lambda_{i}} \frac{\partial \Lambda(\boldsymbol{\lambda})}{\partial \lambda_{j}}\right\}=\frac{1}{\sigma^{2}} \int_{0}^{T} \frac{\partial \tilde{r}_{\lambda}(t)}{\partial \lambda_{i}} \frac{\partial \tilde{r}_{\lambda}(t)}{\partial \lambda_{j}} d t,
$$

for $i \neq j$, where $\lambda_{i}$ is the $i$ th component of $\lambda$, and $[\mathrm{B}]_{i j}$ denotes the element of matrix $\mathrm{B}$ in the $i$ th row and $j$ th column.

From (3), (8) and (9), the FIM can be obtained for various displacement functions. In the following, two different scenarios are considered depending on the available information on the channel coefficients.

\section{A. Case-1: Known Channel Coefficients}

First, we assume that the channel coefficients $\alpha_{\mathrm{b}}$ and $\alpha_{\mathrm{h}}$ are known at the receiver. This scenario approximates the situation in which a number of initial bursts are used for channel estimation, and the estimates for $\alpha_{b}$ and $\alpha_{h}$ obtained using those initial bursts are employed for the remaining estimation period.

For known $\alpha_{\mathrm{b}}$ and $\alpha_{\mathrm{h}}$, the unknown parameter vector $\lambda$ reduces to $\boldsymbol{\lambda}=\left[\begin{array}{ll}\boldsymbol{\theta}_{\mathrm{b}} & \boldsymbol{\theta}_{\mathrm{h}}\end{array}\right]$. Then, from (3), (8) and (9), the FIM can be obtained, after some manipulations, as

$$
\mathbf{I}_{1}=\left[\begin{array}{ll}
\mathbf{I}_{\mathrm{bb}} & \mathbf{I}_{\mathrm{bh}} \\
\mathbf{I}_{\mathrm{bh}}^{T} & \mathbf{I}_{\mathrm{hh}}
\end{array}\right]
$$

where

$$
\left[\mathbf{I}_{\mathrm{bb}}\right]_{i j}=\frac{\alpha_{\mathrm{b}}^{2} \tilde{E}}{N \sigma^{2}} \sum_{k=0}^{N-1} \frac{\partial g_{k}\left(\boldsymbol{\theta}_{\mathrm{b}}\right)}{\partial \theta_{\mathrm{b}, i}} \frac{\partial g_{k}\left(\boldsymbol{\theta}_{\mathrm{b}}\right)}{\partial \theta_{\mathrm{b}, j}}
$$


for $i, j=1, \ldots, K_{1}$,

$$
\left[\mathbf{I}_{\mathrm{hh}}\right]_{i j}=\frac{\alpha_{\mathrm{h}}^{2} \tilde{E}}{N \sigma^{2}} \sum_{k=0}^{N-1} \frac{\partial h_{k}\left(\boldsymbol{\theta}_{\mathrm{h}}\right)}{\partial \theta_{\mathrm{h}, i}} \frac{\partial h_{k}\left(\boldsymbol{\theta}_{\mathrm{h}}\right)}{\partial \theta_{\mathrm{h}, j}}
$$

for $i, j=1, \ldots, K_{2}$, and

$$
\left[\mathbb{I}_{\mathrm{bh}}\right]_{i j}=\frac{\alpha_{\mathrm{b}} \alpha_{\mathrm{h}}}{N \sigma^{2}} \sum_{k=0}^{N-1} \tilde{P}\left(h_{k}\left(\boldsymbol{\theta}_{\mathrm{h}}\right)-g_{k}\left(\boldsymbol{\theta}_{\mathrm{b}}\right)\right) \frac{\partial g_{k}\left(\boldsymbol{\theta}_{\mathrm{b}}\right)}{\partial \theta_{\mathrm{b}, i}} \frac{\partial h_{k}\left(\boldsymbol{\theta}_{\mathrm{h}}\right)}{\partial \theta_{\mathrm{h}, j}}
$$

for $i=1, \ldots, K_{1}$ and $j=1, \ldots, K_{2}$, with $\tilde{R}(x)$ representing the autocorrelation of the first derivative of the pulse burst $w(t)$ in $(2)$; i.e.

$$
\check{R}(x)=\int_{-\infty}^{\infty} w^{\prime}(t+x) w^{\prime}(t) \mathrm{d} t
$$

and $\tilde{E}=\tilde{R}(0)$.

Then, the CRLB for the covariance of an unbiased estimate of $\lambda$ can be expressed as [12]

$$
\operatorname{Cov}\{\hat{\lambda}\} \geq \mathbb{I}_{1}^{-1}
$$

where $\mathbf{B} \geq \mathbf{C}$ means that $\mathbf{B}-\mathbf{C}$ is positive semi-definite.

Let the first elements of $\theta_{\mathrm{b}}$ and $\theta_{\mathrm{h}}$ denote the rate (frequency) parameters to be estimated; i.e., respiration and heartbeat rates, respectively. Then, the CRLBs for estimating $f_{\mathrm{b}}=\theta_{\mathrm{b}, 1}$ and $f_{\mathrm{h}}=\theta_{\mathrm{h}, 1}$ can be obtained, by utilizing the inversion formula for block matrices, as

$$
\begin{aligned}
& \operatorname{Var}\left\{\hat{f}_{\mathrm{b}}\right\} \geq\left[\left(\mathbf{I}_{\mathrm{bb}}-\mathbf{I}_{\mathrm{bh}} \mathbf{I}_{\mathrm{hh}}^{-1} \mathbf{I}_{\mathrm{bh}}^{T}\right)^{-1}\right]_{11}, \\
& \operatorname{Var}\left\{\hat{f}_{\mathrm{h}}\right\} \geq\left[\left(\mathbf{I}_{\mathrm{hh}}-\mathbf{I}_{\mathrm{bh}}^{T} \mathbf{I}_{\mathrm{bb}}^{-1} \mathbf{I}_{\mathrm{bh}}\right)^{-1}\right]_{11},
\end{aligned}
$$

where $\hat{f}_{\mathrm{b}}$ and $\hat{f}_{\mathrm{h}}$ are unbiased estimates for $\hat{f}_{\mathrm{b}}$ and $f_{\mathrm{h}}$, respectively.

If the time-delay between the signal components refecting from the heart and the chest satisfies the condition that $h_{k}\left(\theta_{\mathrm{h}}\right)-g_{k}\left(\theta_{\mathrm{b}}\right)>\Delta_{1} \forall k$ such that $\widetilde{R}(x)=0$ for $x \geq \Delta_{1}$, the FIM in (10) reduces to a block diagonal structure, since it can be shown from (13) that $\mathbb{I}_{b h}=0$. Then, (16) can be expressed as

$$
\operatorname{Var}\left\{\hat{f}_{\mathrm{b}}\right\} \geq\left[\mathbf{I}_{\mathrm{bb}}^{-1}\right]_{11}, \quad \operatorname{Var}\left\{\hat{f}_{\mathrm{h}}\right\} \geq\left[\mathbf{I}_{\mathrm{hh}}^{-1}\right]_{11} .
$$

In $[5]$, the round trip delay diflerence between the signal components reflecting from the skin and the heart is predicted to be around $1.7 \mathrm{~ns}$ based on narrowband models. If the result is close to this value for UWB signals, the bound in (17) is valid for sub-nanosecond UWB pulses, which means that the same accuracy for respiration rate estimation can be achieved as in the case of perfectly known heartbeat related parameters, and vice versa. In other words, unknown parameters of the respiration and heartbeat related signals do not deteriorate the estimation accuracy of each other if UWB pulses with sufficiently short durations are employed.

\section{B. Case-2: Unknown Channel Coefficients}

Now we consider the case in which the channel coefficients for the signal components reflecting from the chest cavity and the heart are considered unknown. In this case, the unknown parameter vector $\lambda$ is given by (5), which consists of $K_{1}$ । $K_{2}+2$ components.

From (3), (8) and (9), the FIM for this case can be obtained, after some manipulation, as

$$
\mathbb{I}_{2}=\left[\begin{array}{lll}
\mathbf{I}_{b b} & \mathbf{I}_{b h} & \mathbf{I}_{b \alpha} \\
\mathbf{I}_{b h}^{T} & \mathbf{I}_{h h} & \mathbf{I}_{h \alpha} \\
\mathbb{I}_{b \alpha}^{T} & \mathbb{I}_{h \alpha}^{T} & \mathbf{I}_{\alpha \alpha \alpha}
\end{array}\right]
$$

where $\mathbb{I}_{b b}, \mathbb{I}_{h h}$ and $\mathbb{I}_{b h}$ are as in $(11)-(13), \mathbb{I}_{b \alpha}$ is a $K_{1} \times 2$ matrix given by

$$
\begin{aligned}
& {\left[\mathbf{I}_{\mathrm{b} \alpha}\right]_{i 1}=-\frac{\alpha_{\mathrm{b}} \hat{E}}{N \sigma^{2}} \sum_{k=0}^{N-1} \frac{\partial g_{k}\left(\boldsymbol{\theta}_{\mathrm{b}}\right)}{\partial \theta_{\mathrm{b}, i}}} \\
& {\left[\mathbf{I}_{\mathrm{b} \alpha}\right]_{k 2}=-\frac{\alpha_{\mathrm{b}}}{N \sigma^{2}} \sum_{k=0}^{N-1} \hat{R}\left(h_{k}\left(\boldsymbol{\theta}_{\mathrm{h}}\right)-g_{k}\left(\boldsymbol{\theta}_{\mathrm{b}}\right)\right) \frac{\partial g_{k}\left(\boldsymbol{\theta}_{\mathrm{b}}\right)}{\partial \theta_{\mathrm{b}, i}}}
\end{aligned}
$$

for $i=1, \ldots, K_{1}, \mathbb{I}_{\text {ho }}$ is a $K_{2} \times 2$ matrix given by

$$
\begin{aligned}
& {\left[\mathbb{I}_{\mathrm{h} \alpha}\right]_{i 1}=-\frac{\alpha_{\mathrm{h}} \hat{E}}{N \sigma^{2}} \sum_{k=0}^{N-1} \frac{\partial h_{k}\left(\boldsymbol{\theta}_{\mathrm{h}}\right)}{\partial \theta_{\mathrm{h}, i}}} \\
& {\left[\mathbb{I}_{\mathrm{h} \alpha}\right]_{i 2}=-\frac{\alpha_{\mathrm{h}}}{N \sigma^{2}} \sum_{k=0}^{N-1} \hat{R}\left(g_{k}\left(\boldsymbol{\theta}_{\mathrm{b}}\right)-h_{k}\left(\boldsymbol{\theta}_{\mathrm{h}}\right)\right) \frac{\partial h_{k}\left(\boldsymbol{\theta}_{\mathrm{h}}\right)}{\partial \theta_{\mathrm{h}, i}}}
\end{aligned}
$$

for $i=1, \ldots, K_{2}$, and $\mathbf{I}_{\alpha \alpha}$ is expressed as

$$
\begin{aligned}
& {\left[\mathbf{I}_{\alpha \alpha}\right]_{11}=\left[\mathbf{I}_{\alpha \alpha}\right]_{22}=\frac{E}{\sigma^{2}}} \\
& {\left[\mathbf{I}_{\alpha \alpha}\right]_{12}=\left[\mathbb{I}_{\alpha \alpha}\right]_{21}=\frac{1}{N \sigma^{2}} \sum_{k=0}^{N-1} R\left(h_{k}\left(\theta_{\mathrm{h}}\right)-g_{k}\left(\theta_{\mathrm{b}}\right)\right)}
\end{aligned}
$$

with $R(x)$ representing the autocorrelation function of the pulse burst $w(t), E=R(0), \hat{R}(x)=\int_{-\infty}^{\infty} w(t) w^{\prime}(t+x) \mathrm{d} t$, and $\hat{E}=\hat{R}(0)$.

In general, the CRLBs for estimating the rate parameters $f_{b}$ and $f_{\mathrm{h}}$ can be obtained as

$$
\operatorname{Var}\left\{\hat{f}_{\mathrm{b}}\right\} \geq\left[\mathbf{I}_{2}^{-1}\right]_{11}, \operatorname{Var}\left\{\hat{f}_{h}\right\} \geq\left[\mathbf{I}_{2}^{-1}\right]_{\left(K_{1}+1\right)\left(K_{1}+1\right)} .
$$

However, under certain conditions, simpler expressions than (22) can be obtained. Note from (19) and (20) that if the delay difference between the signal components reflecting from the heart and the chest cavity satisfies the condition that $h_{k}\left(\theta_{h}\right)-$ $g_{k}\left(\theta_{\mathrm{b}}\right)>\Delta_{2} \forall k$ such that $\hat{R}(x)=0$ for $x \geq \Delta_{2}$, then $\mathbf{I}_{b \alpha}=\mathbf{I}_{\text {h } \alpha}=0$. In that case, (22) reduces to (16) of Case-1. In other words, under the stated condition, the knowledge of the channel coefficients does not affect the theoretical lower bounds.

\section{SUBOPTIMAL SOLUTION}

After obtaining the theoretical limits for respiration and heartbeat rate estimation, this section considers a suboptimal estimator that asymptotically achieves the CRLBs under certain conditions. Note that the ML solution in (7) is an optimal solution; however, it requires correlation of the received signal, over the observation interval $[0, T]$, with a template signal $\tilde{r}_{\lambda}(t)$ for various values of the parameter $\lambda$, which requires, in general, optimization over a $\left(K_{1}+K_{2}+2\right)$-dimensional 


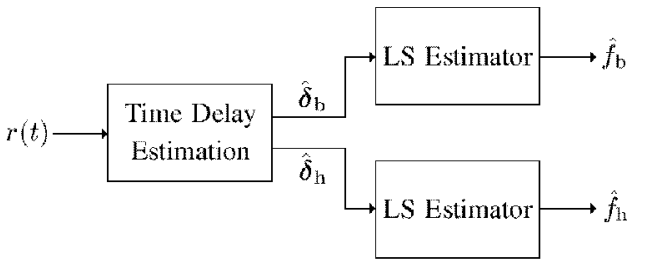

Fig. 2. A two-step suboptimal solution for joint estimation of respiration and heartbeat rates.

space, which has prohibitive complexity for practical implementations.

Due to the complexity of the ML estimator in (7), a twostep suboptimal estimator, as shown in Figure 2, is proposed. This algorithm first performs delay estimation of the signal components due to respiration and heartbeat in each burst, and then estimates the desired signal parameters from the delay estimates in the first step via an LS algorithm.

Assuming that the channel parameters are known at the receiver $^{2,3}$ as in Case-1 of Section III-A, let $\delta_{\mathrm{b}, k} \doteq g_{k}\left(\theta_{\mathrm{b}}\right)$ and $\delta_{h, k} \doteq h_{k}\left(\theta_{h}\right)$ represent the time-delays in the $k$ th burst of the signal components due to respiration and heartbeat, respectively $(k=0,1, \ldots, N-1)$. Note that the estimation of $\delta_{b, k}$ and $\delta_{h, k}$ in a given burst is a special case of multipath delay estimation, studied extensively in the literature [13], [14]. In the first step, the proposed estimator performs ML. estimation of time-delays in each burst [14].

Let the delay estimates related to respiration and heartbeat components obtained in the first step be $\hat{\delta}_{\mathrm{b}}=$ $\left[\hat{\delta}_{\mathrm{b}, 0} \hat{\delta}_{\mathrm{b}, 1} \cdots \hat{\delta}_{\mathrm{b}, N-1}\right]$ and $\hat{\delta}_{\mathrm{h}}=\left[\hat{\delta}_{\mathrm{h}, 0} \hat{\delta}_{\mathrm{h}, 1} \cdots \hat{\delta}_{\mathrm{h}, N-1}\right]$. In the second step, these delay estimates are employed in the following LS estimators to estimate the parameters related to respiration and heartbeat:

$$
\begin{aligned}
& \hat{\theta}_{\mathrm{b}}=\arg \min _{\theta_{\mathrm{b}}} \sum_{k=0}^{N-1}\left(\hat{\delta}_{\mathrm{b}, k}-g_{k}\left(\boldsymbol{\theta}_{\mathrm{b}}\right)\right)^{2}, \\
& \hat{\boldsymbol{\theta}}_{\mathrm{h}}=\arg \min _{\boldsymbol{\theta}_{\mathrm{h}}} \sum_{k=0}^{N-1}\left(\hat{\delta}_{\mathrm{h}, k}-h_{k}\left(\boldsymbol{\theta}_{\mathrm{h}}\right)\right)^{2} .
\end{aligned}
$$

Note that this two-step estimator is usually considerably simpler and more practical than the $\mathrm{ML}$ solution in (7) since it does not have to perform correlations over the whole observation interval for all different values of $\lambda$. Instead, it first performs a search over a two-dimensional space for delay estimates in each burst. Then, it uses these timedelay estimates from different bursts for the LS estimation of the desired parameters. For comparison purposes, it can be observed that for small number of bursts and $K_{1}>K_{2}$, the complexity of the proposed algorithm is dominated by the search over a $K_{1}$ dimensional space, whereas the complexity of the $M L$ algorithm in (7) is determined by the search over a $\left(K_{1}+K_{2}\right)$ dimensional space.

\footnotetext{
${ }^{2}$ In fact, the knowledge of the ratio between the channel parameters, $\alpha_{b} / \alpha_{h}$, is sufficient for the proposed algorithm.

${ }^{3}$ We will see how this assumption can be approximately realized in practical scenarios in Section IV-B.
}

\section{A. Optimality Properties}

Although the proposed estimator is suboptimal in general, it can be shown that it is asymptotically optimal under certain conditions. Towards this end, the following result is helpful:

Lemmat 114]: Consider the signal model

$$
r_{k}(t)=\sum_{i=1}^{L} \alpha_{i} w\left(t-\delta_{i}\right)+\sigma n_{k}(t)
$$

for $t \in\left[0, T_{\mathrm{s}} \mid\right.$, where $n_{h}(t)$ is zero mean white Gaussian noise with unit spectral density, and $\delta_{i}$ 's are the time-delays to be estimated.

The ML estimation of the multipath delays can be obtained by finding $\hat{\delta}_{i}$ 's that maximize $\int_{-\infty}^{\infty} r_{k}(t) \sum_{i=1}^{L} \alpha_{i} w\left(t-\hat{\delta}_{i}\right) \mathrm{d} t$. Then, the ML delay estimates can be modeled, for sufficiently large SNRs, as

$$
\hat{\delta}_{i}=\delta_{i}+\eta_{i}
$$

for $i=1, \ldots$, L, where $\eta=\left[\eta_{1} \cdots \eta_{L}\right]$ is a zero mean multivariate Gaussian random variable with the inverse of its covariance matrix given by

$$
[\Psi]_{i i}=4 \pi^{2} \beta^{2} \mathrm{SNR}_{i}, \quad[\Psi]_{i j}=-\frac{\alpha_{i} \alpha_{j}}{\sigma^{2}} \tilde{R}\left(\delta_{j}-\delta_{i}\right)
$$

for $i \neq j$. Note that in (26), $\tilde{R}(x)$ is as in (14), SNR $E \alpha_{i}^{2} / \sigma^{2}$, with $E$ denoting the energy of $w(t)$, and $\beta$ is effective bandwidh, defined as $\beta^{2}=\int f^{2}|W(f)|^{2} d f / \int|W(f)|^{2} d f$, with $W(f)$ denoting the Fourier transform of $w(t)$.

From Lemma 1, it can be deduced that the ML estimation of the time-delays in a given burst $k$ for the signal components due to respiration and heartbeat can be obtained by correlating the received signal in that burst; i.e., $r(t)$ for $t \in\left[k T_{\mathrm{s}},(k+1) T_{\mathrm{s}}\right]$, with a template signal for various delay values. In addition, the estimation errors can be modeled by a multivariate Gaussian random variable that depends on the signal characteristics and the noise level. Therefore, the delay estimates in the first step of the proposed algorithm can be modeled as

$$
\left[\begin{array}{l}
\hat{\delta}_{\mathrm{b}, k} \\
\hat{\delta}_{\mathrm{h}, k}
\end{array}\right]=\left[\begin{array}{l}
g_{k}\left(\theta_{\mathrm{b}}\right) \\
h_{k}\left(\theta_{\mathrm{h}}\right)
\end{array}\right]+\frac{1}{\sqrt{N}}\left[\begin{array}{l}
\eta_{\mathrm{b}, k} \\
\eta_{\mathrm{h}, k}
\end{array}\right]
$$

where

$$
\left[\begin{array}{l}
\eta_{\mathrm{b}, k} \\
\eta_{h, k}
\end{array}\right] \sim \mathcal{N}\left(\left[\begin{array}{l}
0 \\
0
\end{array}\right],\left[\begin{array}{cc}
4 \pi^{2} \beta^{2} \mathrm{SNR}_{\mathrm{b}} & -\frac{\alpha_{\mathrm{b}} \alpha_{\mathrm{h}}}{\sigma^{2}} \gamma_{k} \\
-\frac{\alpha_{b} \alpha_{h}}{\sigma^{2}} \gamma_{k} & 4 \pi^{2} \beta^{2} \mathrm{SNR} R_{h}
\end{array}\right]^{-1}\right)
$$

for $k=0,1, \ldots, N-1$, with $\mathrm{SNR}_{\mathrm{b}}=E \alpha_{\mathrm{b}}^{2} / \sigma^{2}, \mathrm{SNR}_{\mathrm{h}}=$ $E \alpha_{h}^{2} / \sigma^{2}$ and $\gamma_{h}=\tilde{R}\left(h_{h}\left(\theta_{h}\right)-g_{k}\left(\theta_{b}\right)\right)$.

From (27) and (28), the following result can be obtained. Note that the noise components for different bursts are independent since they are affected from independent noise realizations.

Proposition 1: Consider a set of time-delay measurements $\hat{\delta}=\left[\hat{\delta}_{0} \hat{\delta}_{1} \ldots \hat{\delta}_{N-1}\right]$, where $\hat{\delta}_{k}=\left[\hat{\delta}_{\mathrm{b}, k} \hat{\delta}_{\mathrm{h}, k}\right]^{T}$ is modeled as in (27) and (28) for $k=0,1, \ldots, N-1$. Assume that $h_{k}\left(\theta_{\mathrm{h}}\right)-g_{k}\left(\theta_{\mathrm{b}}\right)>\Delta_{1} \forall k$, with $\tilde{R}(x)=0$ for $x \geq \Delta_{1}$. Then, the CRLB for the covariance matrix of an unbiased estimate of $\left[\theta_{\mathrm{b}} \boldsymbol{\theta}_{\mathrm{h}}\right]$ is the same as the expression in (15), where the 
FIM is given by (10)-(13).

Proof: See Appendix A.

Note that under the assumption in Proposition 1, the ML solution for $\left[\boldsymbol{\theta}_{\mathrm{b}} \boldsymbol{\theta}_{\mathrm{h}}\right]$ can be obtained from (27) and (28) as

$$
\begin{array}{r}
{\left[\hat{\boldsymbol{\theta}}_{\mathrm{b}} \hat{\boldsymbol{\theta}}_{\mathrm{h}}\right]=\arg \min _{\left[\boldsymbol{\theta}_{\mathrm{b}} \boldsymbol{\theta}_{\mathbf{h}}\right]} \sum_{k=0}^{N-1}\left[\alpha_{b}^{2}\left(\hat{\delta}_{\mathrm{b}, k}-g_{k}\left(\boldsymbol{\theta}_{\mathrm{b}}\right)\right)^{2}\right.} \\
\left.+\alpha_{h}^{2}\left(\hat{\delta}_{\mathrm{h}, k}-h_{k}\left(\boldsymbol{\theta}_{\mathrm{h}}\right)\right)^{2}\right],
\end{array}
$$

which is equivalent to the second step of the proposed solution in (23). In other words, the LS solution in the second step is the ML solution for the signal model in (27) and (28). Since the ML estimator asymptotically achieves the CRLB [12], which is equal to the CRLB in Section III according to Proposition 1 , the LS solution in (23) provides an asymptotically optimal solution for estimating $\boldsymbol{\theta}_{\mathrm{b}}$ and $\boldsymbol{\theta}_{\mathrm{h}}$ under the conditions stated in Lemma 1 and Proposition 1.

\section{B. Practical Issues}

Although the proposed estimator in the previous section is asymptotically optimal under certain conditions, it assumes the knowledge of the channel coefficients $\alpha_{\mathrm{b}}$ and $\alpha_{\mathrm{h}}$, which may not be true in many situations. Therefore, in a practical system, the channel coefficients should be estimated from the initial burst(s), which can then be used in the implementation of the proposed approach, since they are assumed to be constant during the observation interval $t \in[0, T]$. In other words, $\alpha_{\mathrm{b}}$ and $\alpha_{\mathrm{h}}$ can be estimated from the received signal $r(t)$ for $t \in\left[0, N_{\mathrm{c}} T_{\mathrm{s}}\right]$ where $N_{\mathrm{c}} \geq 1$ is the number of bursts used for estimation of the channel coefficients.

Since the estimates for the channel coefficients will include error in practice, the proposed algorithm cannot achieve the CRLB in reality. However, for small estimation errors, the performance will still be quite close to the optimal under the conditions stated in Lemma 1 and Proposition 1.

\section{Simulation Results}

In this section, numerical studies and simulations are performed to evaluate the CRLB expressions derived in Section III, and to investigate the performance of the proposed estimator in Section IV.

Consider a system that uses the Gaussian mono-cycle $p(t)=$ $\left(1-\frac{4 \pi t^{2}}{\zeta^{2}}\right) e^{-\frac{2 \pi t^{2}}{\zeta^{2}}} / \sqrt{E_{p}}$, where $E_{p}$ is used to adjust the energy of the pulse in the simulations, and $\zeta$ determines the pulse width $\left(T_{\mathrm{W}} \approx 2.5 \zeta\right)$ [15].

In the simulations, the displacement functions $g_{k}\left(\boldsymbol{\theta}_{\mathrm{b}}\right)$ and $h_{k}\left(\boldsymbol{\theta}_{\mathrm{h}}\right)$ are modeled as

$$
\begin{aligned}
& g_{k}\left(\boldsymbol{\theta}_{\mathrm{b}}\right)=\tau_{\mathrm{b}}+A_{\mathrm{b}} \sin \left(2 \pi f_{\mathrm{b}} k T_{\mathrm{s}}+\phi_{\mathrm{b}}\right), \\
& h_{k}\left(\boldsymbol{\theta}_{\mathrm{h}}\right)=\tau_{\mathrm{h}}+A_{\mathrm{h}} \sin \left(2 \pi f_{\mathrm{h}} k T_{\mathrm{s}}+\phi_{\mathrm{h}}\right) .
\end{aligned}
$$

In Figure 3 and 4, the CRLB expressions in (16) and (22), corresponding to Case- 1 and Case- 2 respectively, are plotted for various SNRs and pulse widths ${ }^{4}$. The system parameters are $T_{\mathrm{s}}=0.1 \mathrm{~s}, f_{\mathrm{b}}=0.5 \mathrm{~Hz}, f_{\mathrm{h}}=1.1 \mathrm{~Hz}, \tau_{\mathrm{b}}=0, \tau_{\mathrm{h}}=1.7$

\footnotetext{
${ }^{4}$ Square-roots of the expressions are plotted and the lower bounds are obtained in units of $\mathrm{Hz}$.
}

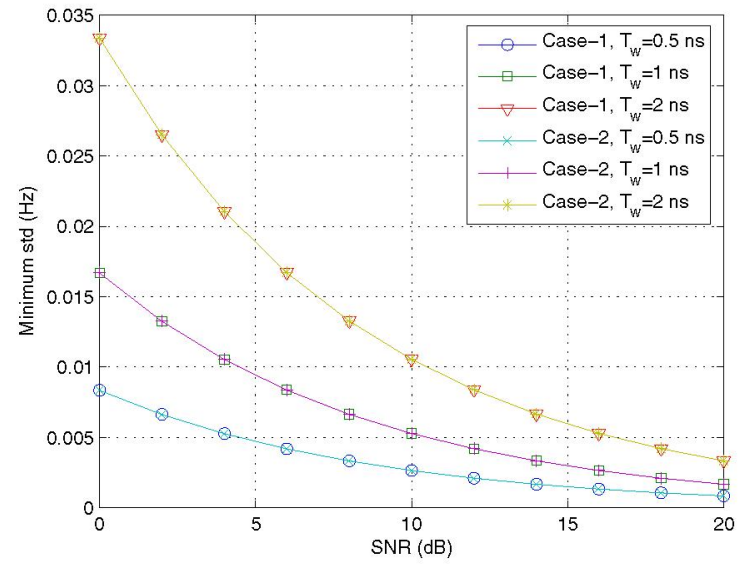

Fig. 3. CRLBs for respiration rate estimation for Case-1 and Case-2.

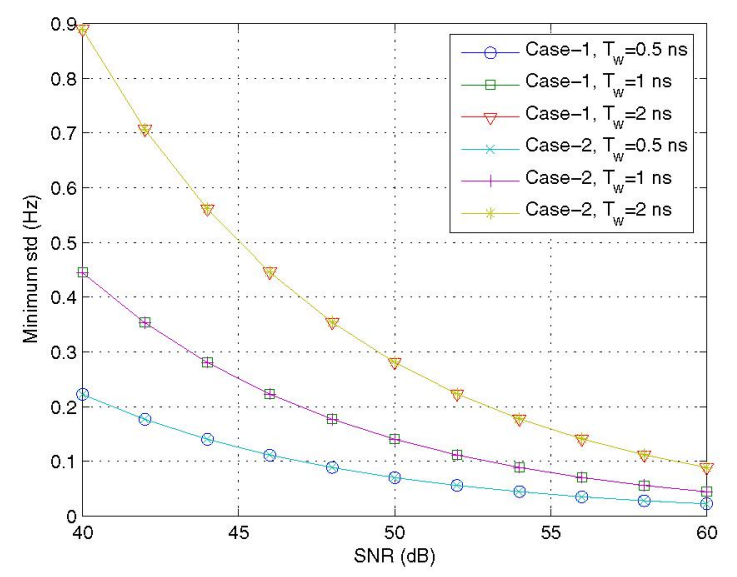

Fig. 4. CRLBs for heartbeat rate estimation for Case- 1 and Case-2.

$\mathrm{ns}, A_{\mathrm{b}}=6.67 \times 10^{-11} \mathrm{~s}, A_{\mathrm{h}}=1.667 \times 10^{-11} \mathrm{~s}, \phi_{\mathrm{b}}=\phi_{\mathrm{h}}=$ $0, \alpha_{\mathrm{b}}=1, \alpha_{\mathrm{h}}=0.0015$ and $N=500$. From the figures, it is observed that the CRLBs for Case- 1 and Case- 2 match very closely for all SNR and $T_{\mathrm{w}}$ values, since the time-delay difference between the respiration and heartbeat components is larger than $1 \mathrm{~ns}$. In addition, the accuracy increases as the pulse width is decreased, which is expected since higher time resolution results in better localization of the object. Also, it is observed that higher SNRs ${ }^{5}$ are needed for reliable heartbeat rate estimation than those needed for reliable respiration rate estimation, which is due to the additional loss (about $28 \mathrm{~dB}$ ) caused by the propagation of the signal from the skin to the heart and back to the skin [5].

In Figure 5, the same parameters as in the previous scenario were employed, and the lower bounds for respiration rate estimation are plotted versus pulse width for various SNRs. It is observed again that as the pulse width decreases, the accuracy of the estimation increases. Moreover, the slope decreases as the SNR increases, which means that for high SNRs, the loss in accuracy when using wider pulses (pulses with smaller bandwidths) is smaller than the loss for low SNRs. Similar observations are made for the CRLB versus pulse width curves for the heartbeat rate estimation (not

\footnotetext{
${ }^{5}$ In order to obtain higher SNR values, longer observation intervals and more pulse combining can be employed.
} 


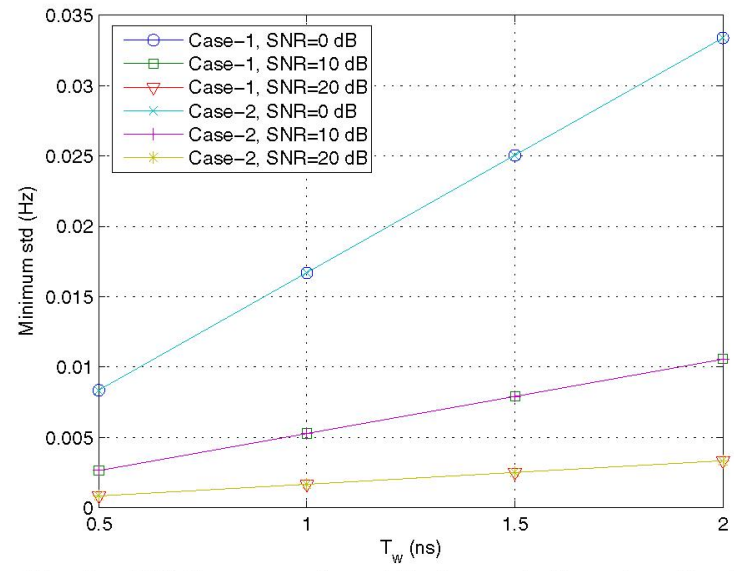

Fig. 5. CRLB versus pulse width for respiration rate estimation.

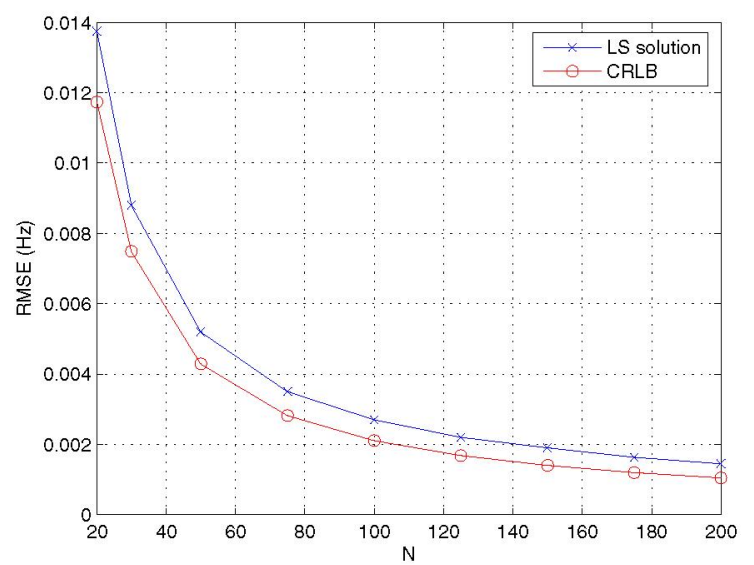

Fig. 6. RMSE versus SNR for the LS solution and the CRLB.

shown).

Finally, Figure 6 compares the performance of the suboptimal LS solution in Section IV with the CRLB for a pulse width of $T_{\mathrm{W}}=0.5 \mathrm{~ns}$ and for an SNR of $20 \mathrm{~dB}$ in a respiration rate estimation scenario. All the parameters are as in the previous scenarios, except that $A_{\mathrm{b}}, A_{\mathrm{h}}, \phi_{\mathrm{b}}$ and $\phi_{\mathrm{h}}$ are assumed to be known, and average values are obtained by generating $f_{\mathrm{b}}, f_{\mathrm{h}}$, $\tau_{\mathrm{b}}$ and $\tau_{\mathrm{h}}$ uniformly from the sets $[0.25,1] \mathrm{Hz},[1,1.5] \mathrm{Hz}$, $[-0.5,0.5]$ ns and $[1,2]$ ns, respectively. From the figure, it is observed that the performance of the LS solution is quite close to the theoretical lower bound.

\section{Conclusions}

Theoretical limits for joint estimation of respiration and heartbeat signal parameters have been studied for IR-UWB systems. Generic CRLB expressions have been obtained for various cases and a suboptimal estimator has been proposed. It has been shown that the proposed estimator is asymptotically optimal under certain conditions.

\section{APPENDIX}

\section{A. Proof of Proposition 1}

From the model given by (27) and (28) for the time-delay estimates, the distribution of $\hat{\boldsymbol{\delta}}=\left[\hat{\boldsymbol{\delta}}_{0} \hat{\boldsymbol{\delta}}_{1} \cdots \hat{\boldsymbol{\delta}}_{N-1}\right]$, with $\hat{\boldsymbol{\delta}}_{k}=$ $\left[\hat{\delta}_{\mathrm{b}, k} \hat{\delta}_{\mathrm{h}, k}\right]^{T}$, for a given $\boldsymbol{\lambda}=\left[\boldsymbol{\theta}_{\mathrm{b}} \boldsymbol{\theta}_{\mathrm{h}}\right]$ can be expressed, under the assumption of $h_{k}\left(\boldsymbol{\theta}_{\mathrm{h}}\right)-g_{k}\left(\boldsymbol{\theta}_{\mathrm{b}}\right)>\Delta_{1}$ (which makes sure that the off-diagonal terms in (28) are zero), as

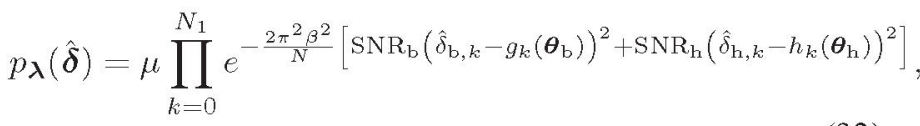

where $\mu$ is a constant independent of $\lambda$.

From (32), the elements of the FIM $\tilde{\mathbf{I}}$ can be obtained from

$$
[\tilde{\mathbf{I}}]_{i i}=\mathrm{E}\left\{\left(\frac{\partial \log p_{\boldsymbol{\lambda}}(\hat{\boldsymbol{\delta}})}{\partial \lambda_{i}}\right)^{2}\right\}, i=1, \ldots, K_{1}+K_{2},
$$

and

$$
[\tilde{\mathbf{I}}]_{i j}=\mathrm{E}\left\{\frac{\partial \log p_{\boldsymbol{\lambda}}(\hat{\boldsymbol{\delta}})}{\partial \lambda_{i}} \frac{\partial \log p_{\boldsymbol{\lambda}}(\hat{\boldsymbol{\delta}})}{\partial \lambda_{j}}\right\}, \quad i \neq j .
$$

From the definition of the effective bandwidth, it can be shown that $4 \pi^{2} \beta^{2} \mathrm{SNR}_{\mathrm{b}}=\tilde{E} \alpha_{\mathrm{b}}^{2} / \sigma^{2}$ and $4 \pi^{2} \beta^{2} \mathrm{SNR}_{\mathrm{h}}=$ $\tilde{E} \alpha_{\mathrm{h}}^{2} / \sigma^{2}$. Then, from (32), (33) and (34) can be calculated, after some manipulation ${ }^{6}$, and it can be shown that $\tilde{\mathbf{I}}=$ $\left[\begin{array}{ll}\mathbf{I}_{\mathrm{bb}} & \mathbf{I}_{\mathrm{bh}} \\ \mathbf{I}_{\mathrm{bh}}^{T} & \mathbf{I}_{\mathrm{hh}}\end{array}\right]$. In other words, $\tilde{\mathbf{I}}$ is equal to $\mathbf{I}_{1}$ in Section III-A, which completes the proof.

\section{REFERENCES}

[1] U. S. Federal Comm. Commission, FCC 02-48: First Report and Order.

[2] S. Gezici, Z. Tian, G. B. Giannakis, H. Kobayashi, A. F. Molisch, H. V. Poor and Z. Sahinoglu, "Localization via ultra-wideband radios," IEEE Signal Processing Magazine, vol. 22, issue 4, pp. 70-84, July 2005.

[3] M. Z. Win and R. A. Scholtz, "Impulse radio: How it works," IEEE Communications Letters, 2(2): pp. 36-38, Feb. 1998.

[4] S. Venkatesh, C. R. Anderson, N. V. Rivera, and R. M. Buehrer, "Implementation and analysis of respiration-rate estimation using impulsebased UWB," IEEE Military Commun. Conf. (MILCOM), vol. 5, pp. 3314-3320, Oct. 17-20, 2005.

[5] E. M. Staderini "UWB radars in medicine," IEEE Aerospace and Electronic Magazine, vol. 17, no. 1, pp. 13-18, Jan. 2002.

[6] Y. Chen, E. Gunawan, K. S. Low, Y. Kim, C. B. Soh, A. R. Leyman and L. L. Thi, "Non-invasive respiration rate estimation using ultrawideband distributed cognitive radar system," Proc. 28th IEEE EMBS Annual International Conference, pp. 920-923, Aug. 30-Sep. 3, 2006.

[7] S. Gezici and Z. Sahinoglu, "Theoretical limits for estimation of vital signal parameters using impulse radio UWB," IEEE International Conference on Communications (ICC 2007), June 24-27, 2007.

[8] G. Ossberger, T. Buchegger, E. Schimback, A. Stelzer and R. Weigel, "Non-invasive respiratory movement detection and monitoring of hidden humans using ultra wideband pulse radar," IEEE Int. Workshop on Ultra Wideband Systems (IWUWBS'04), pp. 395-399, May 18-21, 2004.

[9] M. Nowogrodzki, D. D. Mawhinney and H. F. Milgazo, "Noninvasive microwave instruments for the measurement of respiration and heart rates," Proc. IEEE National Aerospace and Electronics Conference (NAECON 1984), pp. 958-960, vol. 2, May 21, 1984.

[10] O. B. Lubecke, P. W. Ong and V. M. Lubecke" "10 GHz Doppler radar sensing of respiration and heart movement," Proc. IEEE 28th Annual Northeast Bioengineering Conference, pp. 55-56, April 20-21, 2002.

[11] M. A. Richards, Fundamentals of Radar Signal Processing, 1st ed. New York: McGraw-Hill, 2005.

[12] H. V. Poor, An Introduction to Signal Detection and Estimation, 2nd ed. New York: Springer-Verlag, 1994.

[13] P. J. Ianniello, "Large and small error performance limits for multipath time delay estimation," IEEE Transactions on Acoustics, Speech, and Signal Processing, vol. ASSP-34, no. 2, pp. 245-251, Apr. 1986.

[14] Y. Qi, H. Kobayashi and H. Suda, "On time-of-arrival positioning in a multipath environment," IEEE Transactions on Vehicular Technology, vol. 55, issue 5, pp. 1516-1526, Sep. 2006.

[15] F. Ramirez-Mireles and R. A. Scholtz, "Multiple-access performance limits with time hopping and pulse-position modulation," Proc. IEEE Military Commun. Conf. (MILCOM), vol. 2, pp. 529-533, Oct. 1998.

${ }^{6}$ The details are omitted due to the space limitation. 